\title{
Surgical reconstructive treatment for infraosseous peri-implantitis defects with a submerged healing approach: A prospective controlled study
}

\author{
Shih-Cheng Wen $^{1,2}$ ｜ Shayan Barootchi ${ }^{3}$ (c) | Wen-Xia Huang ${ }^{4} \quad$ Hom-Lay Wang ${ }^{3}$ (i)
}

${ }^{1}$ Taipei Medical University, Taipei, Taiwan

${ }^{2}$ Taipei County, Taiwan, ROC

${ }^{3}$ Department of Periodontics \& Oral Medicine, University of Michigan School of Dentistry, Ann Arbor, MI

${ }^{4}$ Periodontics Department, Xiamen Stomatological Hospital, Xiamen, China

\section{Correspondence}

Hom-Lay Wang, Department of Periodontics and Oral Medicine, University of Michigan School of Dentistry, 1011 North University Avenue, Ann Arbor, MI 481091078, USA.

Email:homlay@umich.edu

\begin{abstract}
Background: The aim of this study was to assess the reconstructive potential of a submerged healing approach for the treatment of infraosseous peri-implantitis defects.

Methods: Patients with a diagnosis of peri-implantitis were recruited. Implant suprastructures were removed before the surgical treatment, which included implant surface and defect detoxification using implantoplasty, air-power driven devices, and locally delivered antibiotics. The augmentation procedure included a composite bone graft and a non-resorbable membrane followed by primary wound coverage and a submerged healing of 8 months, at which point membranes were removed, and peri-implant defect measurements were obtained as the primary outcome. Secondary endpoints included assessment of cone-beam computed tomography (CBCT) and probing depth (PD) reductions.

Results: Thirty implants in 22 patients were treated. A significant clinical bone gain of $3.22 \pm 0.41 \mathrm{~mm}$ was observed at 8 months. Radiographic analysis also showed an average gain of $3.47 \pm 0.41 \mathrm{~mm}$. Three months after installment of new crowns, final PD measures showed a significant reduction compared to initial examinations and a significant reduction in bleeding on probing compared to examinations at the pre-surgical visit.

Conclusions: Reconstruction of infraosseous peri-implantitis defects is feasible with thorough detoxification of implant sites, and a submerged regenerative healing approach.
\end{abstract}

\section{KEYWORDS}

bone regeneration, dental implants, implant failure, peri-implantitis, periodontal diseases, surgical treatment

\section{1 | INTRODUCTION}

Peri-implantitis is an inflammatory response that leads to the irreversible loss of implant supporting bone. This condition and is clinically characterized by an implant site that exhibits signs of inflammation in addition to radiographic bone loss beyond initial biological bone remodeling. ${ }^{1}$ Studies have estimated that peri-implantitis affects approximately one in every four individuals $(22 \%)$ with dental implants, ${ }^{2,3}$ and is the leading cause of implant failure.$^{4,5}$

With the emergence of this disease, a variety of therapeutic options have been set forward for its management. 
Non-surgical therapies alone have generally shown to be inadequate for treating moderate and severe forms of peri-implantitis.$^{6-9}$ Surgical therapies such as open flap debridement and resective procedures can provide a greater access to the peri-implant defect for enhanced debridement and detoxification, as well as for obtaining probing depth (PD) reduction. ${ }^{7,10}$ Nonetheless, these have also been documented with varying levels of success ,$^{10-12}$ and with failure rates of as high as $\approx 60 \% .{ }^{13}$ Moreover, in spite of achieving disease resolution, because peri-implantitis is an irreversible phenomenon, the implant site after treatment remains devoid of its original supporting bony structures.

More recently, surgical therapies in combination with regenerative protocols have been employed, with a range of biomaterials, adopting many of the principles of guided bone regeneration (GBR) (i.e., use of bone grafting materials and barrier membranes). ${ }^{14,15}$ The aim, aside from resolution of disease, has been to reconstruct the lost periimplant supporting bone.$^{14,16,17}$ To achieve this aim, two main approaches have been used throughout the literature; the submerged approach wherein the implant suprastructures (i.e., crowns) are removed and a primary closure and coverage of the grafted area is obtained, allowing for undisturbed wound healing, and the non-submerged approach wherein removal of the suprastructure is not performed and hence the implant cannot be completely submerged.

Currently, most of the literature on reconstructive treatment for peri-implantitis involves a non-submerged healing approach.${ }^{18-21}$ Indeed, the removal of an implant crown adds extra steps, and accompanies additional time and costs for both the patient and the clinician. Nevertheless, it can be speculated that removal of the implant suprastructures can provide an increased access to the implant surface for enhanced detoxification, and that a submerged healing would ultimately create a more favorable environment for bone formation to occur. Additionally, the prosthesis itself at times may be a contributor to the progression and/or initiation of the disease ${ }^{22}$ Thus, even with successful bone regeneration of a peri-implant defect, adjustment or re-fabrication of a prosthetic component may be necessary for maintaining the stability of the treatment outcomes. Thus, the primary aim of this study was to evaluate the reconstructive potential, in terms of direct clinical bone gain measurements for a submerged regenerative approach when treating infraosseous peri-implantitis defects, as well as secondarily to evaluate changes in radiographic bone levels and peri-implant PDs after installment of new crowns.

\section{2 | MATERIALS AND METHODS}

\subsection{Study registration, design, and participants}

The current study was designed to evaluate the reconstructive potential of a submerged healing approach for the surgical treatment of peri-implantitis. From June of 2017 to 2019, patients with a diagnosis of peri-implantitis ${ }^{1}$ in at least one posterior dental implant who had been on maintenance care and at least a biannual professional cleaning at a university/private practice were assessed for possible recruitment. The study was conducted at a private office in Taipei, Taiwan. The diagnosis of peri-implantitis was based on the definition from the 2017 World Workshop ${ }^{1}$ as a dental implant with bleeding and/or suppuration upon probing, and increased PD measurements compared to previous examinations, in combination with radiographic bone loss beyond initial biological bone remodeling. When lacking previous data, the diagnosis was based on the presence of bleeding and/or suppuration on probing, with periimplant PD measurements of $6 \mathrm{~mm}$ or more, with at least $3 \mathrm{~mm}$ of radiographic bone loss.

The study protocol was in full accordance with the Declaration of Helsinki of 1965, as revisited in Tokyo in 2013 and approved by the by the Institutional Review Board and the local ethical committee (Stomatological Hospital of Xiamen Medical College, \#18950051616). The current article is prepared following the items presented in the STROBE statement (www.strobe-statement.org, checklist provided as a supplementary file).

\section{2 | Eligibility criteria}

The following criteria were applied for patients with a diagnosis of peri-implantitis to be treated as part of this study: (1) a minimum age of 18 years old, (2) systematically healthy or with only a mild to moderate but well-controlled systemic disease, (3) bone-level titanium implants with a vertical defect (of at least $3 \mathrm{~mm}$ ) and surrounding bony walls (i.e., a crater-like defects).

The exclusion criteria comprised: (1) patients with an uncontrolled/untreated systemic or periodontal disease, (2) subjects on medications known to modify bone metabolism or those that could interfere with normal wound healing, (3) patients on recent antibiotic therapy (within the past 2 months), (4) pregnant, or patients planning to get pregnant, (5) smoking $>10$ cigarettes a day, (6) unable to maintain an adequate oral hygiene (O'Leary plaque index $>50 \%$ ) ${ }^{23}$ (7) mobile dental implants, (8) 


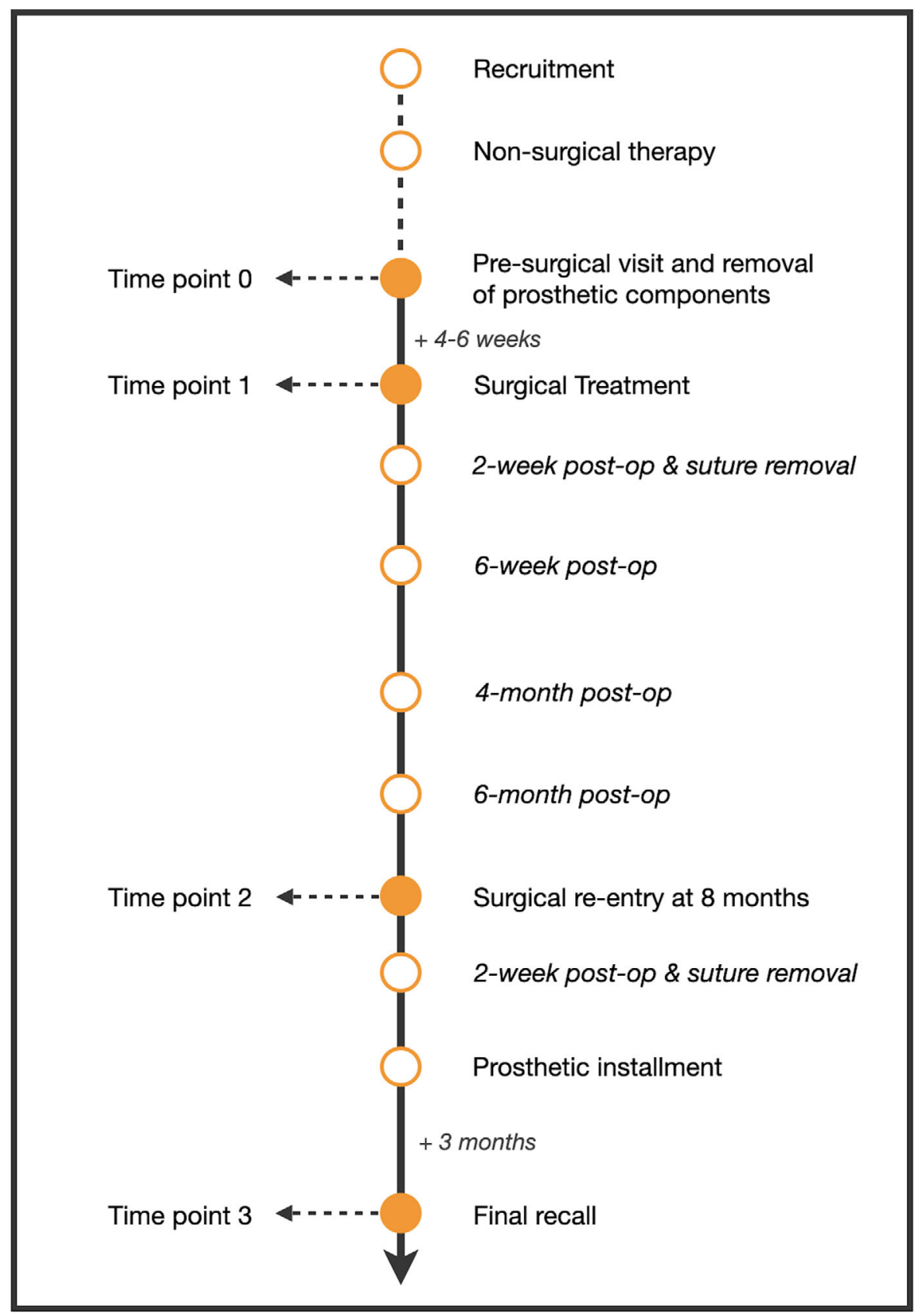

F I G U R E 1 Research timeline demonstrating each study visit from recruitment until the final appointment

implants with defects located outside the bony housing, and other contraindications for undergoing a dental surgery.

After successful fulfillment of the inclusion criteria, details of the study protocol were explained to interested participants, followed by obtaining an informed consent. All recruited subjects also underwent localized non-surgical mechanical debridement (scaling and root planing) on the infected implants, as well as reinforcement of oral hygiene measures at least 1 month before the surgical visit (Figure 1).

\subsection{Clinical evaluation at the pre-surgical appointment (time point 0 ), and at the final recall (time point 3 )}

The following clinical parameters were obtained by a single pre-calibrated study member (S-C.W) at the pre-surgical appointment immediately before removal of implant suprastructures, and at the final recall (3 months after installment of new crowns): (1) Peri-implant PD measurements assessed at six sites (disto-buccal, buccal, mesio-buccal, mesio-lingual, lingual/palatal, 
disto-lingual) from the mucosal margin to the depth of the probable peri-implant pocket with a standard periodontal probe* and recorded in millimeter, (2) presence of bleeding on gentle probing (BOP) assessed dichotomously as either yes/no within 30 seconds of gentle probing; (3) suppuration (SUP) on probing assessed dichotomously (yes/no) after probing, and (4) plaque index (PI) (scores of 0 to 3$).^{23}$

\section{4 | Study protocol}

\subsection{1 | Pre-surgical appointment (time point $0)$}

The prosthetic superstructures (implant crowns) were removed 4 to 6 weeks before the surgical treatment for all implants to undergo regenerative therapy, followed by placement of cover screws, and a localized superficial mechanical debridement. Patients were informed that newly fabricated crowns would be provided after the treatment as part of the study. During this visit, patients were also re-instructed and emphasized on maintaining proper at-home oral hygiene measures with a soft-bristled toothbrush for minimizing trauma to marginal soft tissues, which was also reinforced at each subsequent follow-up interval.

\subsection{2 | Surgical procedure (time point 1)}

All patients received an established treatment protocol by a single experienced surgeon (S-C.W) under local anesthesia as described in a previous report. ${ }^{24}$ Briefly, an intrasulcular incision was performed around the implants for elevation of a full-thickness mucoperiosteal flap on the buccal and lingual/palatal aspects. Vertical releasing incisions were performed as needed, at a distance of at least one tooth/implant away from the surgical site. Next, the peri-implant defects were mechanically debrided and thoroughly degranulated with periodontal curettes ${ }^{\dagger}$.

In case that an implant or a defect did not meet the inclusion criteria (e.g., non-contained defect, or not feasible for regeneration), the implant/patient was to be excluded from the study and treated with an apicallypositioned flap approach or an open flap-debridement procedure.

\footnotetext{
* PCP-UNC 15; Hu-Friedy, Chicago, IL.
}

${ }^{\dagger}$ Gracey curettes; Hu-Friedy, Chicago, IL.

\subsection{3 $\quad$ Defect measurements}

Vertical measurements of the peri-implant osseous defects were obtained using a periodontal probe $\|$ at four sites (buccal, lingual/palatal, mesial, distal) from the most coronal aspect of the implant platform until the depth of the defect (highest point of bone-to-implant contact) at each site.

\subsubsection{Implant detoxification}

Implantoplasty was performed on the exposed threads with rotary instruments ${ }^{\ddagger}$ under copious irrigation, followed by use of an air-abrasive device with glycine powders ${ }^{\S}$, and the application of a locally delivered antimicrobial agent for 5 minutes (Tetracycline, $250 \mathrm{mg}$ and $2.5 \mathrm{cc}$ ).

\subsection{5 | Augmentation procedure}

Multiple perforations were then made on the cortical bone with a $1 / 4$ round bur. A titanium reinforced nonresorbable dense Polytetrafluoroethylene (dPTFE) membran was trimmed to an appropriate size and shape to completely cover the implant sites and adapted and stabilized lingually with fixation screws ${ }^{* *}$. Around 10 to 20 holes were also created on top of the dPTFE membrane with an explorer to encourage blood supply on top of the ridge area. Next, a composite bone graft with a combination of $\approx 60 \%$ freeze-dried bone cortical allograft ${ }^{\dagger \dagger}, 20 \%$ mineralized bovine bone ${ }^{\$+}$, and $20 \%$ autogenous bone (collected using a bone scraper from the adjacent ramus or maxillary tuberosity) was used to completely fill the peri-implant defects. Finally, the membrane was stabilized on the buccal side with at least two fixation screws to ensure a complete and stable coverage of the grafted area. Care was taken to ensure that the membrane was resting on bone and not in contact with adjacent teeth.

\subsection{6 | Primary wound closure}

Periosteal-releasing incisions were performed to allow passive flap advancement and tension-free displacement. Monofilament non-resorbable sutures ${ }^{\S \S}$ were used for flap adaptation with horizontal mattress and simple interrupted and/or modified horizontal mattress sutures for

\footnotetext{
¥ Meisinger, Hager \& Meisinger, Neuss, Germany

$\S$ AirFlow, EMS, Nyon, Switzerland

** Master Pins, Osteogenics Biomedical, USA

${ }^{\dagger \dagger}$ Maxgraf, Botiss, Zossen, Germany

蛙 Cerabone, Botiss, Zossen, Germany

$\S \S 4$-0, Cytoplast-PTFE suture, Osteogenics Biomedical, USA
} 
completely covering the augmented site, with the implants fully submerged.

\subsection{7 | Post-operative instructions and interim recall appointments}

Detailed post-operative instructions were provided to all patients, as well as prescriptions for oral systemic antibiotics (500 mg of amoxicillin three times a day for 10 days, or $250 \mathrm{mg}$ of Zithromax 6 tablets total /sig. Two tablets the 1st day once daily until gone), and analgesic medication (600 mg of ibuprofen, taken as needed). Patients were also recommended to rinse twice daily with a chlorhexidine-containing mouth rinse $(0.12 \%$ Chlorhexidine mouth rinse) for the first 2 weeks.

Patients were initially recalled at 2 weeks for suture removal, and then at 6 weeks, 4 months, and 6 months before the 8-month re-entry for professional oral hygiene instructions, assessment of soft tissue healing and possible complications. Subsequently, all participants were enrolled in a 3-month maintenance program, of which the initial 3-month recall after installation of the new crowns served as the final study time point (time point 3 ) for performing the clinical measurements and re-assessing all treated sites.

\subsection{8 | Re-entry procedure and replacement of suprastructures (time point 2 )}

After 8 months of uninterrupted healing, patients returned for the re-entry procedure and removal of the nonresorbable membranes and titanium screws. After membrane removal, the augmented sites were evaluated, and the same defect measurements taken during the surgical procedures were performed to obtain clinical changes in peri-implant bone levels. Implant cover screws were replaced by appropriate healing abutments, followed by single interrupted sutures for re-adaptation of the flap. Patients returned in 2 weeks for suture removal. During this period, new crowns were designed, fabricated and delivered to all patients and for all treated implants.

\subsubsection{Final recall (time point 3)}

Three months after installment of new implant crowns, at the initial post-surgical maintenance recall, PD measurements were obtained as performed at the pre-surgical visit (time point 0 ). Figure 2 demonstrates the study protocol with an example of a treated case as part of this study.

\section{5 | Outcome measures}

\subsection{1 | Primary outcome}

The primary endpoint of this study was to assess the amount of clinical linear bone gain after the surgical reconstructive treatment (time point 1) until the 8-month reentry (time point 2), through direct measurements of the peri-implant osseous defects at all implant sites. This was termed the clinical vertical defect fill (DF), and assessed at each implant site (buccal, lingual/palatal, mesial, distal) as follows:

$\mathrm{DF}=($ Measurement at the surgical treatment $)-($ Measurement at re-entry procedure)

The average changes at the 4 implant sites were calculated to present the implant score reflecting the average clinical bone gain per treated implant.

\subsection{2 | Secondary outcomes}

\section{2. 1. Radiographic bone gain}

Two identical cone-beam computed tomography (CBCT) scans $^{* * *}$ were obtained following manufacturer instructions (90 kv, $3.2 \mathrm{~mA}, 15 \mathrm{~s}, 685 \mathrm{mGy} . \mathrm{cm} 2$, Voxel size: $150 \mu \mathrm{m}$ $\times 150 \mu \mathrm{m} \times 150 \mu \mathrm{m}$ ) from all patients at time point 0 (after removal of suprastructures), and at time point 2 (before the surgical re-entry) to evaluate the changes in radiographic bone levels at 4 implant sites. Similar to the direct clinical measurements of the peri-implant defects, the highest point of each implant platform served as the reference for the measurements, until the first visible bone-to-implant contact. All radiographic assessments were performed by a calibrated radiologist (C-Y.W), after initial calibration sessions, and an intra-examiner reproducibility rate of at least $85 \%$ on randomly selected scans. Extra attention was paid during this process to ensure reproducible measurements for each scan, by identification of at least three distinct anatomical landmarks on each CBCT slice (sagittal/crosssectional) to serve as references between time points.

Measurements were performed identically at both time points to assess the changes at each peri-implant site to comprise the vertical radiographic defect fill (RDF) per implant sits:

$\mathrm{RDF}=$ (Measurement at the per-surgical appointment) - (Measurement at 8 months)

Similar to the DF, the changes for the four peri-implant sites were averaged to present the implant score relative to RDF.

\footnotetext{
*** Carestream Dental LLC, CS 8100 3D, Atlanta, USA
} 

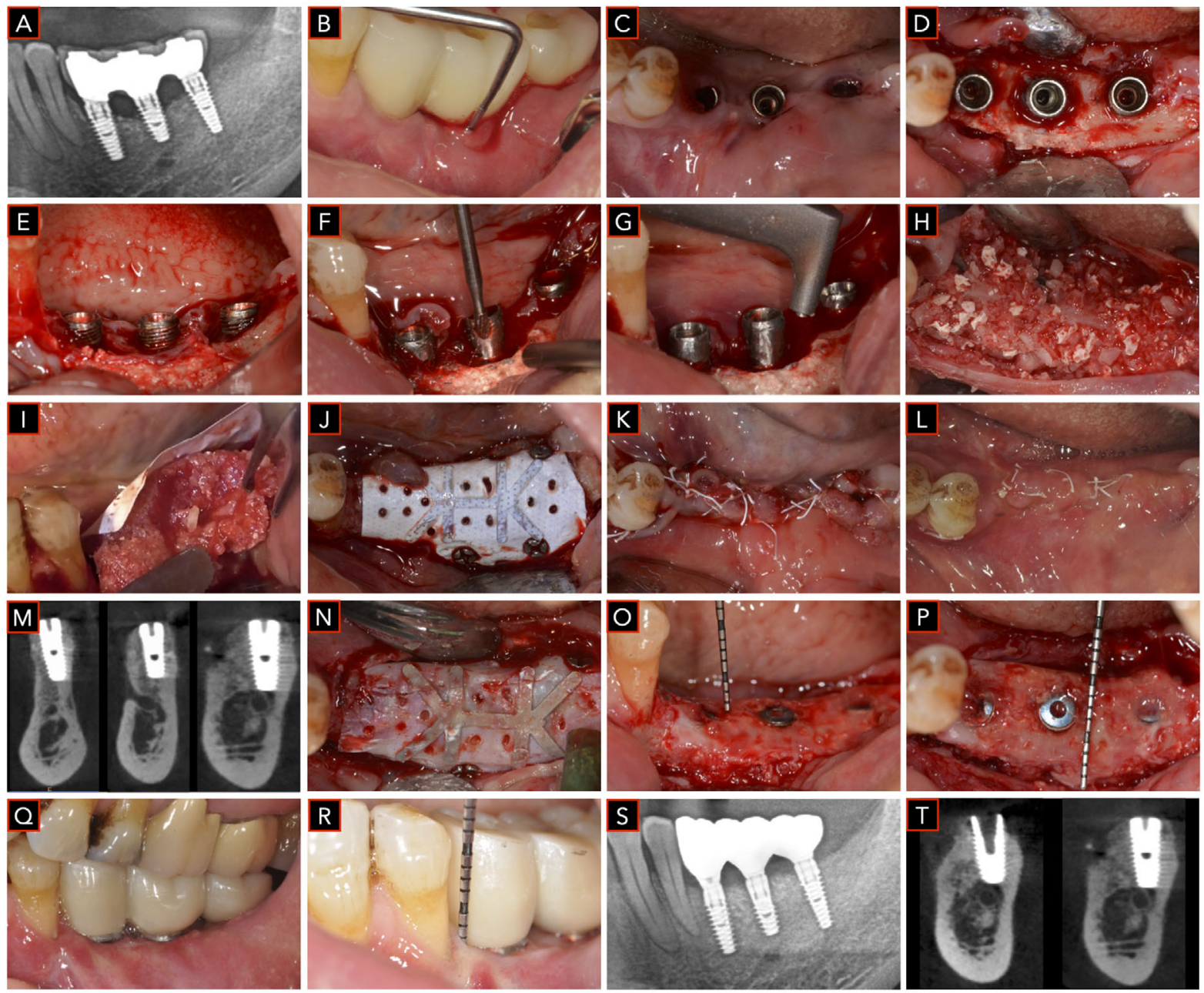

F I G U R E 2 An example of a treated peri-implantitis case in the current study from initiation up to completion of the study. (A and $\mathbf{B}$ ) radiographic and clinical representation of the infected implants before removal of the suprastructures. (C) immediately after removal of suprastructures. (D and E) Occlusal and buccal view of the infected implants after flap elevation. (F) Implantoplasty procedure. (G) Use of an air-abrasive device for debridement of peri-implant defects. (H and $\mathbf{I})$ Augmentation procedure with the application of a composite bone graft material and a perforated non-resorbable barrier membrane. (J) Fixation of the membrane using titanium screws on the buccal and lingual aspect. (K) Immediately after flap closure and obtaining a primary wound coverage. (L) At the two-week post-op before suture removal. (M) Cross-sectional slices of the cone-beam computed tomography scan at the three treated implants during the 8-month recall. (N) Surgical re-entry procedure at 8 months for removing the non-resorbable membrane followed by obtaining defect measurements. (O and $\mathbf{P})$ Buccal, and occlusal views of the regenerated peri-implant bone. (Q) After re-fabrication and installment of new implant prostheses. (R) At the 3-month visit following installment of new prosthesis and obtaining final clinical measurements. (S) Two-dimensional radiographic representation of the treated implants 3 months after placement of new prosthetic suprastructures. (T) Coronal/cross-sectional CBCT slice before- (left, at time point 0 ), and after-regenerative therapy (right, at time point 2 ) of the most distal implant in the presented case

\section{2. 2. Peri-implant $P D$ and $B O P$ reduction}

Changes in PD from time point 0 (before removal of suprastructures) to time point 3 (final recall) were calculated to obtain the PD reduction at each of the six measured peri-implant sites as follows:

PD reduction $=($ Measurement at pre-surgical appointment) - (Measurements at the final recall)

The average changes of the six peri-implant sites were also obtained for descriptive purposes.

BOP was assessed dichotomously (Yes/No) at both time points, for any implant that presented with a positive BOP at any of the six probable sites, and expressed as a percentage of the total treated implants at the specific time point.

\subsection{Outcome assessment and statistical analysis}

Means and standard deviations (SD) were calculated for continuous outcomes (DF, RDF, PD) to descriptively present the gathered clinical data at each time point. BOP was assessed dichotomously and expressed as a percentage 
of implants with a site of bleeding at time point 0 , and at the final recall (time point 3). Linear mixed-regression models were used for statistical assessment of changes in the primary outcome of DF, as well as the secondary outcomes of $\mathrm{RDF}$, and $\mathrm{PD}$ with respect to time and treatment (8 months for DF and RDF, and 12 months for PD). Random effects were included for patient, for implant within patients, and for the implant site of measurement per implant in patients (three-way interaction) to account for repeated measures (multiple implants in some patients, and multiple measurement sites per each implant), along with a fixed effect for time. Model assumptions were tested. The coefficients that present the rate of change for each outcome with respect to time were recorded. Confidence intervals (CIs) were produced, and a $P$ value of 0.05 was set for statistical significance. The average changes in DF, RDF, and PD reduction (per implant) were also obtained for descriptive purposes.

The data management and statistical analyses were performed by a separate author with experience in statistical methodology, who had not participated in any of the measurements or collection of data (S.B), with a specified software $^{\dagger+\dagger}$, and the statistical packages lme $4,{ }^{25}$ and dplyr. ${ }^{26}$

\section{3 | RESULTS}

\subsection{Population and implant characteristics}

Twenty-two patients with a total of 30 implant fixtures were included and treated in this study. The population sample included 12 males and 10 females with an average age of $56 \pm 13.14$ years. All implants were bone level with various implant systems, located in the posterior region (22 molar sites, and eight premolars), and had been in function for at least 2 years before the diagnosis of peri-implantitis. Twenty-two of the treated implants were located in the mandibular region, and eight in the maxilla. Six patients had more than one implant treated, whereas the rest all had only a single implant that was treated. Seven patients reported the use of tobacco, which was $<5$ cigarettes/day, and 18 patients had had a history of periodontal disease and/or treatment for a periodontal condition at some point in the past. The characteristics of the treated sample are presented in Table 1.

All participants completed the study without any dropouts, or implant extractions. Patients were compliant to the prescribed medications and the study followup recalls, and aside from two cases of delayed (beyond 2 weeks) membrane exposure, no unexpected or major

\footnotetext{
$\stackrel{\dagger}{\dagger}$ Rstudio Version 1.1.383, Rstudio, Inc., MA, USA
}

TA B LE 1 Characteristics of the treated sample at baseline

\begin{tabular}{|c|c|c|}
\hline Category & Characteristic & Value \\
\hline \multirow[t]{5}{*}{ Patient } & Male (n, \%) & $12,55 \%$ \\
\hline & Female (n, \%) & $10,45 \%$ \\
\hline & Age $($ mean $\pm S D)$ & $56.8 \pm 13.14$ years \\
\hline & $\begin{array}{l}\text { Smokers }(<5 \\
\quad \text { cigarettes/day) }(\mathrm{n}, \%)\end{array}$ & $7,31.8 \%$ \\
\hline & $\begin{array}{l}\text { Past history of } \\
\text { periodontal disease or } \\
\text { treatment }(\mathrm{n})\end{array}$ & 18 patients \\
\hline \multirow[t]{6}{*}{ Implant } & Maxillary $\operatorname{arch}(\mathrm{n}, \%)$ & $8,22 \%$ \\
\hline & Mandibular $\operatorname{arch}(\mathrm{n}, \%)$ & $22,73 \%$ \\
\hline & Premolar sites $(\mathrm{n}, \%)$ & $8,22 \%$ \\
\hline & Molar sites (n, \%) & $22,73 \%$ \\
\hline & $\begin{array}{l}\text { Screw-retained } \\
\quad \text { restoration }(\mathrm{n}, \%)\end{array}$ & $15,50 \%$ \\
\hline & $\begin{array}{l}\text { Cement-retained } \\
\text { restoration }(n, \%)\end{array}$ & $15,50 \%$ \\
\hline
\end{tabular}

$\mathrm{n}$ corresponds to the number of patients/implants based on the category presented in the left column; SD, standard deviation.

complications had occurred. The two incidence of membrane exposure were both small areas (less than about $5 \mathrm{~mm}^{2}$ ) that did not present with suppuration or discharge and the patients that had noticed the exposure were instructed to rinse with a $0.2 \%$ chlorhexidine mouth rinse, and apply a chlorhexidine-soaked cotton swab twice daily onto the area, until they were removed at 6 weeks.

\section{2 | Primary outcome of direct measurements of linear bone gain after 8 months of submerged healing}

The change rates based on the mixed model for each clinical measure between the two respective time points are presented in Table 2, and the average gain/reduction of the clinical parameters is shown in Table 3. Figure 3 presents clinical and radiographic images from pre-treatment until the follow-up time point of three cases that were treated as part of this study.

At the time of the surgical treatment, the average clinical measurements of the peri-implant osseous defects were $3.37 \mathrm{~mm} \pm 1.88,2.95 \pm 1.66,3.48 \pm 1.60 \mathrm{~mm}$, and $3.63 \pm$ 1.85 on the buccal, lingual/palatal, mesial, and distal sites, respectively. During the surgical re-entry, the obtained measurements for the same peri-implant sites were $0.16 \pm$ $1.86,0.15 \pm 1.66,-0.17 \pm 1.86$, and $0.4 \pm 1.36 \mathrm{~mm}$, respectively (negative sign indicates bone overgrowth relative to the implant platform).

According to the mixed model (Table 2), all changes were statistically significant and amounted to 
TA B L E 2 Results of the mixed-effects regression analysis demonstrating the rates of change for the assessed clinical parameters between the two time points

\begin{tabular}{|c|c|c|c|c|c|c|}
\hline Parameter & Implant site & $\begin{array}{l}\text { Coefficient } \\
(\mathrm{mm})\end{array}$ & SE & $\begin{array}{l}95 \% \\
\text { CIs(Lower, } \\
\text { Upper bound) }\end{array}$ & $P$ value & $\begin{array}{l}\text { Assessed time } \\
\text { points }\end{array}$ \\
\hline \multirow{4}{*}{$\begin{array}{l}\text { Clinical vertical defect } \\
\text { measurements }\end{array}$} & Buccal & -3.321 & 0.25 & $-3.82,-2.81$ & $<0.001$ & \multirow{4}{*}{$\begin{array}{l}\text { Time points } 1 \\
\text { and } 2\end{array}$} \\
\hline & Lingual & -2.803 & 0.25 & $-3.31,-2.28$ & $<0.001$ & \\
\hline & Mesial & -3.656 & 0.27 & $-4.19,-3.11$ & $<0.001$ & \\
\hline & Distal & -3.226 & 0.27 & $-3.77,-2.68$ & $<0.001$ & \\
\hline \multirow{3}{*}{$\begin{array}{l}\text { Radiographic vertical } \\
\text { defect measurements }\end{array}$} & Lingual & -2.993 & 0.27 & $-3.53,-2.45$ & $<0.001$ & \multirow{3}{*}{$\begin{array}{l}\text { Time points } 0 \\
\text { and } 2\end{array}$} \\
\hline & Mesial & -3.836 & 0.27 & $-4.38,-3.28$ & $<0.001$ & \\
\hline & Distal & -3.603 & 0.27 & $-4.15,-3.04$ & $<0.001$ & \\
\hline \multirow{3}{*}{$\begin{array}{l}\text { Peri-implant probing } \\
\text { depth measures }\end{array}$} & Mesio-buccal & -2.966 & 0.29 & $-3.55,-2.37$ & $<0.001$ & \multirow{3}{*}{$\begin{array}{l}\text { Time points } 0 \\
\text { and } 3\end{array}$} \\
\hline & Lingual & -2.766 & 0.33 & $-3.43,-2.10$ & $<0.001$ & \\
\hline & Mesio-lingual & -2.733 & 0.26 & $-3.25,-2.21$ & $<0.001$ & \\
\hline
\end{tabular}

Abbreviation: mm, millimeter; SE, standard error; CIs, Confidence intervals.

Note that changes for clinical and radiographic vertical defect measurements, negative coefficients represent gain in the observed outcomes, whereas for probing depth measures negative scores represent reduction in scores. .

TA B LE 3 Changes in the clinical outcomes per implant according to different time points

\begin{tabular}{|c|c|c|c|c|c|}
\hline \multirow[b]{2}{*}{ Outcome } & \multicolumn{4}{|c|}{ Study time point } & \multirow[b]{2}{*}{ Changes* } \\
\hline & Time point 0 & Time point 1 & Time point 2 & Time point 3 & \\
\hline $\mathrm{DF}(\mathrm{mm} \pm \mathrm{SD})$ & & $3.36 \pm 1.74$ & $0.13 \pm 1.69$ & & $3.22 \pm 0.41$ \\
\hline $\mathrm{RDF}(\mathrm{mm} \pm \mathrm{SD})$ & $3.79 \pm 1.66$ & & $0.31 \pm 1.75$ & & $3.47 \pm 0.41$ \\
\hline BOP $(\%)$ & $100 \%$ & & & $36.6 \%$ & $63.3 \%$ \\
\hline
\end{tabular}

Abbreviations: mm, millimeter; SD, standard deviation.

DF, clinical vertical defect fill reported as the average of the four peri-implant sites.

$\mathrm{RDF}$, radiographic vertical defect fill reported as the average of the four peri-implant sites.

$\mathrm{PD}$, peri-implant probing depth reported as the average of the six peri-implant sites.

BOP, bleeding on probing assessed dichotomously per implant for any of the six peri-implant site that presented with bleeding at the time of assessment. Changes in BOP are represent percentage in reduction.

*Note that changes for DF, and RDF are calculated through subtraction of the initial time point from the secondary time point.

Changes in PD are calculated convey reduction scores as subtraction of the secondary time point from the initial time point.

$3.21 \pm 1.3 \mathrm{~mm}$ in clinical bone gain on the buccal, $2.8 \pm$ $1.4 \mathrm{~mm}$ on the lingual/palatal, $3.66 \pm 1.4 \mathrm{~mm}$ on the mesial, and $3.23 \pm 1.4 \mathrm{~mm}$ on the distal aspects. The average gain for all implant sites amounted to $3.22 \pm 0.41 \mathrm{~mm}$ (Table 3).

\section{3 | Radiographic bone gain}

The initial radiographic (CBCT) measurements of the defects after crown removal showed $4.03 \pm 1.58,3.22 \pm 1.8$,
$3.89 \pm 1.56$, and $4.01 \pm 1.70 \mathrm{~mm}$ on average for the periimplant sites of buccal, lingual/palatal, mesial, and distal, respectively. The corresponding measurements of the four implant sites at the 8-month re-entry demonstrated measurements of $0.55 \pm 1.64,0.22 \pm 1.86,0.05 \pm 1.89$, and $0.4 \pm 1.62 \mathrm{~mm}$, respectively. The resulting changes, based on the mixed model amounted to RDF of $3.48 \pm$ $1.5 \mathrm{~mm}$ on the implants' buccal sides, $2.99 \pm 1.48 \mathrm{~mm}$ on the lingual/palatal aspects, followed by $3.84 \pm 1.5 \mathrm{~mm}$, and $3.6 \pm 1.5 \mathrm{~mm}$ on the mesial and distal sites, respectively. 


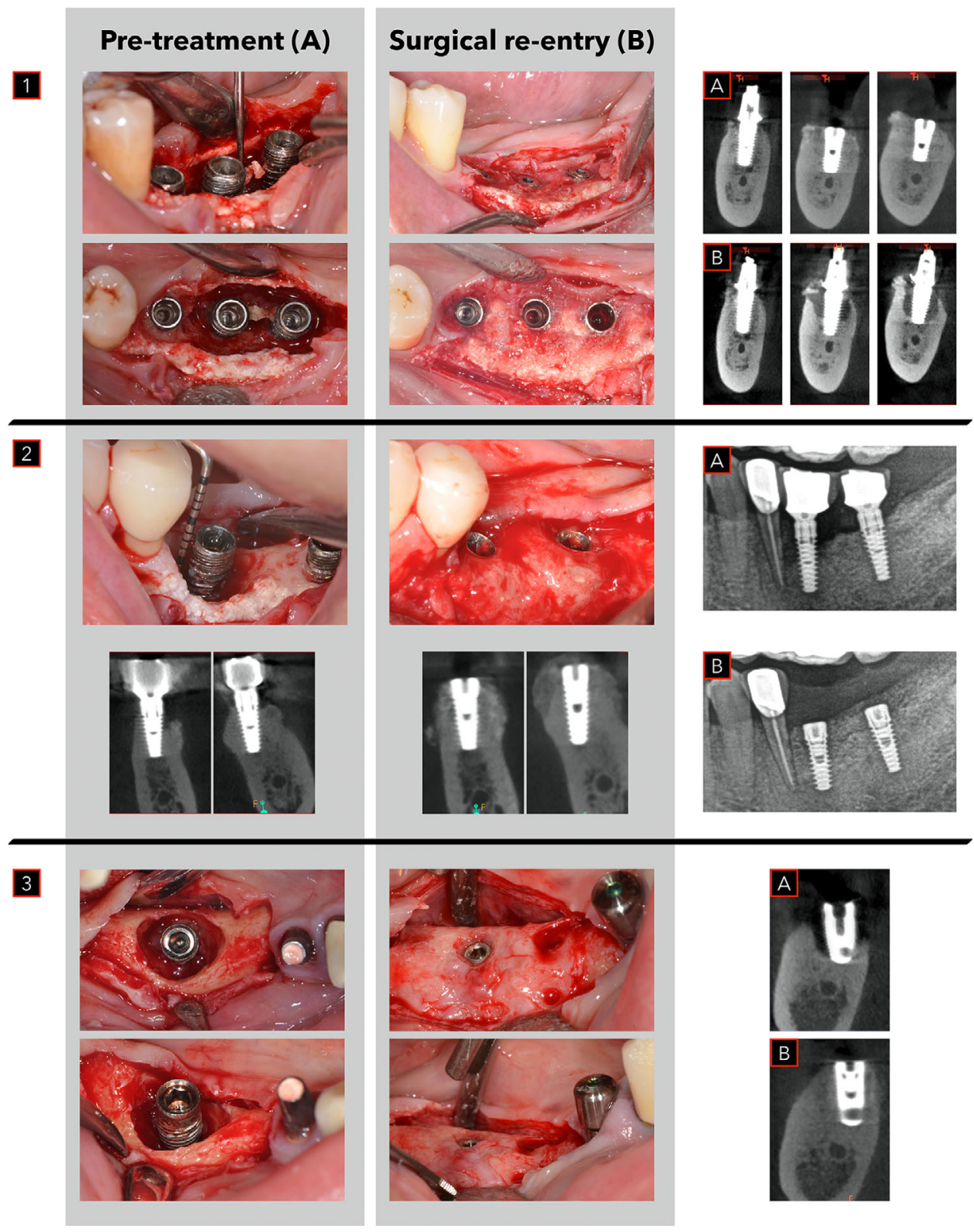

F I G U R E 3 Clinical and radiographic images from pre-treatment until the follow-up time point of three treated cases as part of this study. Note that images with A markings display baseline (pre-treatment) representation of defects, whereas B illustrate the outcomes after 8 months of submerged regenerative healing

All changes presented with statistical significance ( $P$ values $<0.001$, Table 2), and an overall implant score of 3.47 $\pm 0.41 \mathrm{~mm}$ for $\mathrm{RDF}$ (Table 3 ).

\section{4 | Peri-implant PD reduction and bleeding on probing}

Initial PD measurements before the treatment averaged to $5.83 \pm 1.42,6.10 \pm 1.52,6.07 \pm 1.2,5.83 \pm 1.58 \mathrm{~mm}$, $5.70 \pm 1.6$, and $5.33 \pm 1.56 \mathrm{~mm}$ on the mesio-buccal, buccal, disto-buccal, disto-lingual, lingual, and mesiolingual sides, respectively. At the final study recall, 3 months after installment of new crowns, the corresponding values averaged to $2.87 \pm 1.11,2.97 \pm 1.1,3.03 \pm$ $1.19,3.03 \pm 1.03,2.93 \pm 1.34$, and $2.60 \pm 0.89 \mathrm{~mm}$, respectively.

Overall, a significant PD reduction was observed at the final recall for all treated sites as a result of the combined surgical and prosthetic therapy, amounting $2.97 \pm 1.6,3.13$ $\pm 1.6,3.03 \pm 1.5,2.81 \pm 1.4,2.76 \pm 1.8$, and $2.73 \pm 1.4 \mathrm{~mm}$ ( $P$ values $<0.001$, Table 2 ), and an average implant PD reduction score of $2.93 \pm 0.25 \mathrm{~mm}$ (Table 3). In addition, a significant reduction in BOP from $100 \%$ to $36.6 \%$ was also observed from the pre-surgical visit (time point 0 ) until the final recall (time point 3). 
The date pertaining to all investigated outcomes, at their respective time points are also further presented in Table S1.

\section{4 | DISCUSSION}

In the present study, we observed a considerable improvement in all clinical measurements, and a significant reconstruction of infraosseous peri-implantitis defects with the submerged healing approach.

When discussing the treatment of peri-implantitis, a prerequisite for obtaining successful outcomes, is management of its etiology. In part, this entails thorough debridement of the peri-implant defect, proper decontamination of the implant surface, and removal of potential contributing factors to the peri-implant diseases. In addition, removal of implant suprastructures before the surgical procedure, allowed for a greater access to the osseous defects, and implant surfaces at the time of detoxification. Replacement of cover screws at least 1 month before the regenerative therapy also led to the expansion of adjacent soft tissues before the procedure, which is an advantage on its own for such bone augmentation procedures.$^{27}$ Last, in the prospect of obtaining long-term success and avoiding treatment relapse, carefully re-fabricated restorations were provided to all patients as part of the study.

The choice for our selected biomaterials, relative to the application of a non-resorbable membrane stemmed from its wide and predictable use in regenerative procedures .${ }^{28-30}$ One of the corner stones of a successful bone regenerative therapy is the ability to maintain the augmented space with a stable biomaterial, ${ }^{31,32}$ in which blood clot formation, angiogenesis, and eventual bone regeneration can occur ${ }^{31,33}$ This, along with other properties of dPTFE membranes ${ }^{34-37}$ fortified our decision towards selection of this biomaterial for the regenerative protocol. In addition, small perforations were also made on the membranes to aid in blood supply and minimize the possibility of membrane exposure. Indeed, only two incidences of a minor membrane exposure were observed, which presented without purulence and the membranes were removed later on, without an apparent compromise to the regenerative outcomes.

Relative to our choice of bone grafting material, we sought to obtain a cell-containing biomaterial filler capable of triggering osteoblastic migration and differentiation, growth factor release, and formation of new mineralized tissues, without the need for excessive autogenous harvesting/scraping, as well as one that would exhibit a lower biodegradability to serve as a longer lasting scaffold ${ }^{38-40}$ to maintain the augmented space over the intended 8month period. Although to the best of our knowledge no other study on the treatment of peri-implantitis reports the application of such mixture, similar combinations, and with varying ratios of autogenous and allogenic/xenogenic bovine bone have been used for horizontal and vertical ridge augmentation in the oral cavity. ${ }^{41-43}$

Thus far, most of the human research on the reconstructive treatment of peri-implantitis has involved the use of a non-submerged approach, ${ }^{18-21,44}$ and the few studies that have used a submerged approach, have assessed slightly different outcomes or at alternative time points.$^{14,45,46} \mathrm{At}$ this moment, no other human study has strictly assessed the outcomes of a submerged healing for peri-implantitis through direct clinical examinations of bone fill, a virtue of the necessity to remove the non-resorbable membrane and fixation screws, that we defined as our primary outcome. Therefore, a direct comparison of our results, in particular our primary endpoint to the literature may not be feasible.

Schwarz et al. was the first to conduct a comparison of the submerged and non-submerged approach in an animal model ${ }^{47}$. The authors concluded that although both groups obtained significant improvements in clinical parameters, the implants in the submerged group achieved better outcomes, and showed greater re-osseointegration potential ${ }^{47}$. Roos-Jansåker et al. in 12 patients with periimplantitis-affected Brånemark implants, used a submerged protocol, ${ }^{46}$ and radiographically assessed the changes in bone levels, which averaged to $2.3 \mathrm{~mm}$ in defect fill (ranging from 1 to $5.1 \mathrm{~mm}$ ) at 6 months. Interestingly the same group had also conducted a previous study that included a non-submerged regenerative group and had obtained less favorable results. ${ }^{48}$ The authors had concluded that the undisturbed wound healing by a submerged approach may have led to the differences in their regenerative outcomes.

A recent randomized clinical trial comparing two bioabsorbable membranes (collagen, and concentrated growth factor) with a bovine bone for a submerged protocol, reported a mean $\mathrm{RDF}$ of $1.98 \pm 0.7$, and $1.63 \pm 1 \mathrm{~mm}$ in either groups 12 months after the reconstructive treatment (obtained through averaging mesial and distal peri-apical radiographic bone levels). ${ }^{14}$ The authors had also reported a significant BOP reduction of 77 to $80 \%$ in both treatment arms at 6 months, ${ }^{14}$ which is slightly higher than our findings 3 months after installment of new implant crowns. This difference may be attributable to a more stringent BOP assessment that was applied in our study, as even a single BOP-positive site per six, would render the implant BOP-positive, contrary to the study by Isler et al.

In a more recent study, Monje et al. treated 27 implants in 15 patients with peri-implantitis using a submerged healing approach.$^{45}$ Relative to the augmentation, the authors used a mixture of an autogenous and xenogenic bovine bone with the application of an absorbable 
collagen membrane, and assessed the outcomes with periapical radiographs at 1 year, reporting a significant gain of $2.2 \mathrm{~mm}$ (ranging 0 to $8.6 \mathrm{~mm}$ ) in marginal bone levels, and $3.7 \mathrm{~mm}$ reduction in PD (0.7 to 5.9). ${ }^{45}$ The many design-driven differences, and baseline characteristics of the referenced report relative to ours (the chosen biomaterials, outcome assessment, initial defect characteristics, refabrication of all crowns, etc.) could have led to a greater gain in the mean mesial/distal radiographic scores in our study $(3.72 \pm 1.41 \mathrm{~mm})$, and the lower PD reductions (2.93 $\pm 0.25 \mathrm{~mm}$ ).

Despite the advantage that can be obtained with a submerged healing approach, readers should bear in mind that removal of the implant suprastructure, especially weeks before the regenerative procedure, can be challenging for many to accept, in particular when re-fabrication of a crown may be indicated (such as cement retained prosthesis, or overly contoured restorations). This portion of the treatment, although indeed beneficial, can also accompany certain challenges. Aside from patients' acceptance, or the clinician's own motivation towards this additional step, it should also be considered that achieving a primary wound closure in such cases is technique sensitive, and the required flap release is also likely to accompany a greater post-operative patient morbidity. Therefore, future randomized studies in the area of reconstructive peri-implantitis therapy are needed to quantify the added value of a submerged healing protocol with suprastructure removal, and its cost-effectiveness compared to a nonsubmerged approach, or one wherein temporary removal of suprastructures are done merely to facilitate decontamination.

In the current study it was our aim to evaluate the potential reconstructive capacity of confined infraosseous peri-implantitis defects. Therefore, our results should be interpreted with caution as not all peri-implantitis defects may be favorable for regeneration. Although confirmation of defect morphology and the degree of bone loss was obtained after flap reflection and granulation tissue removal, careful examination of pre-surgical CBCT scans (at time point 0) allowed for this preliminary assessment, especially as crown removal had led to enhanced radiographic images and less scatter radiation.

Indeed, the lack of a non-submerged treatment arm, different types of bone level implants, or a control group relative to the biomaterial selection (grafting materials, barrier membranes) may limit the generalizability of our results. Nonetheless, as data pertaining to the submerged healing approach is scarce in the literature, and the treatment of peri-implantitis is a challenging task to accomplish, we sought to use a gold standard protocol with the most chance of success in our view. Furthermore, it must be mentioned that true and definitive regeneration can only be assessed through histological biopsies, which was not performed in our study. Lastly, we deem necessary future randomized studies to explore other facets relative to the reconstructive aspect, as well as long-term follow-up investigations in the context of possible treatment relapse, and the ability of the reconstructed bone to stably support the treated implants under occlusal forces.

\section{5 | CONCLUSION}

Considering the limitations of this study, it can be concluded that a submerged healing approach for the surgical reconstructive treatment of peri-implantitis can lead to significant reconstruction of the lost peri-implant supporting bone. This can be attributed to the merits provided by the removal of the implant suprastructures that allow for enhanced peri-implant defect debridement and implant surface detoxification, as well as the possibility to obtain a primary coverage and undisturbed wound healing throughout the regenerative process.

\section{ACKNOWLEDGMENTS}

The authors express their gratitude to Chung-Yu Wu (Medical Radiologist, Shih-Hwa Dental Implant Center, Taipei County, Taiwan) for performing the radiographic measurements.

\section{CONFLICT OF INTEREST}

The authors declare no potential conflict of interest with respect to publication of this report or the enlisted and materials within this manuscript.

\section{FUNDING INFORMATION}

The authors do not have any financial interests, either directly nor indirectly, in the products or information listed in the paper.

\section{AUTHOR CONTRIBUTIONS}

Shih-Cheng Wen: Conception and design of the study; performed the surgical procedures, initial and final drafting of the work; Final approval of the version to be published; accountable for all aspects of the work.

Shayan Barootchi: Design of the study, acquisition, and interpretation of data and analyses, manuscript preparation and the initial draft, final reviewal of the work; accountable for all aspects of the work. 
Wen-Xia Huang: Conception and study design, contribution to manuscript writing, critical review of the final draft, accountable for all aspects of the work.

Hom-Lay Wang: Design of the study; critical review of the draft and contribution to the writing of the manuscript; Final approval of the version to be published and accountable to the accuracy or integrity of the work.

\section{DATA AVAILABILITY STATEMENT}

The data that support the findings of this study are available from the corresponding author upon request.

\section{OR C ID}

Shayan Barootchi @ https://orcid.org/0000-0002-5347-

6577

Hom-Lay Wang (D) https://orcid.org/0000-0003-4238-1799

\section{RE F E RE N C ES}

1. Berglundh T, Armitage G, MG, et al. Peri-implant diseases and conditions: Consensus report of workgroup 4 of the 2017 World Workshop on the Classification of Periodontal and Peri-Implant Diseases and Conditions. J Periodontol 2018;89(suppl 1):S313S318.

2. Derks JC, Peri-implant health and disease. A systematic review of current epidemiology. J Clin Periodontol 2015;42(suppl 16):S158-171

3. Mombelli A, Muller N, N, The epidemiology of peri-implantitis. Clin Oral Implants Res 2012;23(suppl 6):67-76.

4. Ramseier CA, Eick S, Bronnimann C, Buser D, Bragger U, GE, Host-derived biomarkers at teeth and implants in partially edentulous patients. A 10-year retrospective study. Clin Oral Implants Res 2016;27:211-217.

5. Zitzmann NU, T, Definition and prevalence of peri-implant diseases. J Clin Periodontol 2008;35:286-291.

6. Suarez-Lopez Del Amo F, Yu SH, HL, Non-surgical therapy for peri-implant diseases: a systematic review. J Oral Maxillofac Res 2016;7:e13.

7. Faggion CM, Jr, Chambrone L, Listl S, YK, Network metaanalysis for evaluating interventions in implant dentistry: the case of peri-implantitis treatment. Clin Implant Dent Relat Res 2013;15:576-588

8. Lindhe J, Meyle J, Group DoEWoP. Peri-implant diseases: consensus Report of the Sixth European Workshop on periodontology. J Clin Periodontol 2008;35:282-285.

9. Barootchi S, Ravida A, Tavelli L, Wang HL. Nonsurgical treatment for peri-implant mucositis: a systematic review and metaanalysis. Int J Oral Implantol (Berl) 2020;13:123-139.

10. Carcuac O, Derks J, Abrahamsson I, Wennstrom JL, Petzold M, Berglundh T. Surgical treatment of peri-implantitis: 3-year results from a randomized controlled clinical trial. J Clin Periodontol 2017;44:1294-1303.

11. Hallstrom H, Persson GR, Lindgren S, Renvert S. Open flap debridement of peri-implantitis with or without adjunctive systemic antibiotics: a randomized clinical trial. J Clin Periodontol 2017;44:1285-1293.

12. Schwarz F, John G, Schmucker A, Sahm N, Becker J. Combined surgical therapy of advanced peri-implantitis evaluating two methods of surface decontamination: a 7-year follow-up observation. J Clin Periodontol 2017;44:337-342.

13. de Waal YC, Raghoebar GM, Meijer HJ, Winkel EG, van Winkelhoff AJ. Prognostic indicators for surgical peri-implantitis treatment. Clin Oral Implants Res2016;27:1485-1491.

14. Isler SC, Soysal F, Ceyhanli T, Bakirarar B, Unsal B. Regenerative surgical treatment of peri-implantitis using either a collagen membrane or concentrated growth factor: a 12-month randomized clinical trial. Clin Implant Dent Related Res 2018;20:703-712.

15. Larsson L, Decker AM, Nibali L, Pilipchuk SP, Berglundh T, Giannobile WV. Regenerative medicine for periodontal and peri-implant diseases. J Dental Res 2016;95:255-266.

16. Aghazadeh A, Rutger Persson G, Renvert S. A single-centre randomized controlled clinical trial on the adjunct treatment of intra-bony defects with autogenous bone or a xenograft: results after 12 months. J Clin Periodontol 2012;39:666-673.

17. Renvert S, Roos-Jansaker AM, Persson GR. Surgical treatment of peri-implantitis lesions with or without the use of a bone substitute-a randomized clinical trial. J Clin Periodontol 2018;45:1266-1274.

18. Galarraga-Vinueza ME, Obreja K, Magini R, Sculean A, Sader $\mathrm{R}$, Schwarz F. Volumetric assessment of tissue changes following combined surgical therapy of peri-implantitis: a pilot study. J Clin Periodontol 2020.

19. Daugela P, Cicciu M, Saulacic N. Surgical regenerative treatments for peri-implantitis: meta-analysis of recent findings in a systematic literature review. J Oral Maxillofac Res 2016;7:e15.

20. de Tapia B, Valles C, Ribeiro-Amaral T, et al. The adjunctive effect of a titanium brush in implant surface decontamination at peri-implantitis surgical regenerative interventions: a randomized controlled clinical trial. J Clin Periodontol 2019;46:586-596.

21. Jepsen K, Jepsen S, Laine ML, et al. Reconstruction of periimplant osseous defects: a multicenter randomized trial. J Dental Res 2016;95:58-66.

22. Katafuchi M, Weinstein BF, Leroux BG, Chen YW, Daubert DM. Restoration contour is a risk indicator for peri-implantitis: a cross-sectional radiographic analysis. J Clin Periodontol 2018;45:225-232.

23. O'Leary TJ, Drake RB, Naylor JE. The plaque control record. $J$ Periodontol 1972;43:38.

24. Wen S-CH, W-X; Wang, H-L. Regeneration of peri-implantitis infrabony defects: report on three cases. Int $J$ Periodontics Restorat Dent 2019;39.

25. Bates DM, M; Bolker, B; Walker, S. Fitting linear mixed-effects models using lme4. J Statis Software 2015;67:1-48.

26. Wickham HF, R; Henry, L, Müller K., dplyr: A Grammar of Data Manipulation. 2019.

27. Byun SH, Kim SY, Lee H, et al. Soft tissue expander for vertically atrophied alveolar ridges: prospective, multicenter, randomized controlled trial. Clin Oral Implants Res 2020;31:585-594.

28. Barber HD, Lignelli J, Smith BM, Bartee BK. Using a dense PTFE membrane without primary closure to achieve bone and tissue regeneration. J Oral Maxillofacial Surge 2007;65:748-752.

29. Wen SC, Barootchi S, Huang WX, Wang HL. Time analysis of alveolar ridge preservation using a combination of mineralized bone-plug and dense-polytetrafluoroethylene membrane: a histomorphometric study. J Periodontol 2020;91:215-222.

30. Urban IA, Monje A, Lozada JL, Wang HL. Long-term evaluation of peri-implant bone level after reconstruction of severely 
atrophic edentulous maxilla via vertical and horizontal guided bone regeneration in combination with sinus augmentation: a case series with 1 to 15 years of loading. Clin Implant Dent Related Res 2017;19:46-55.

31. Wang HL, Boyapati L. "PASS" principles for predictable bone regeneration. Implant Dent 2006;15:8-17.

32. Simion M, Jovanovic SA, Trisi P, Scarano A, Piattelli A. Vertical ridge augmentation around dental implants using a membrane technique and autogenous bone or allografts in humans. Int $J$ Periodontics Restorative Dent 1998;18:8-23.

33. Sigurdsson TJ, Hardwick R, Bogle GC, Wikesjo UM. Periodontal repair in dogs: space provision by reinforced ePTFE membranes enhances bone and cementum regeneration in large supraalveolar defects. J Periodontol 1994;65:350-356.

34. Carbonell JM, Martin IS, Santos A, Pujol A, Sanz-Moliner JD, Nart J. High-density polytetrafluoroethylene membranes in guided bone and tissue regeneration procedures: a literature review. Int J Oral Maxillofacial Surge 2014;43:75-84.

35. Walters SP, Greenwell H, Hill M, Drisko C, Pickman K, Scheetz JP. Comparison of porous and non-porous teflon membranes plus a xenograft in the treatment of vertical osseous defects: a clinical reentry study. J Periodontol 2003;74:1161-1168.

36. Laurito D, Cugnetto R, Lollobrigida M, et al. Socket Preservation with d-PTFE membrane: histologic analysis of the newly formed matrix at membrane removal. Int J Periodontics Restorative Dent 2016;36:877-883.

37. Urban IA, Lozada JL, Jovanovic SA, Nagursky H, Nagy K. Vertical ridge augmentation with titanium-reinforced, densePTFE membranes and a combination of particulated autogenous bone and anorganic bovine bone-derived mineral: a prospective case series in 19 patients. Int J Oral Maxillofacial Implants 2014;29:185-193.

38. Galindo-Moreno P, Hernandez-Cortes P, Aneiros-Fernandez J, et al. Morphological evidences of Bio-Oss(R) colonization by CD44-positive cells. Clin Oral Implants Res 2014;25:366-371.

39. Sanz M, Dahlin C, Apatzidou D, et al. Biomaterials and regenerative technologies used in bone regeneration in the craniomaxillofacial region: consensus report of group 2 of the 15th European Workshop on Periodontology on Bone Regeneration. JClin Periodontol 2019;46(Suppl 21):82-91.

40. Zhang X, Tiainen H, Haugen HJ. Comparison of titanium dioxide scaffold with commercial bone graft materials through micro-finite element modelling in flow perfusion. Med Biological Eng Computing 2019;57:311-324.

41. Ronda M, Rebaudi A, Torelli L, Stacchi C. Expanded vs. dense polytetrafluoroethylene membranes in vertical ridge augmentation around dental implants: a prospective randomized con- trolled clinical trial. Clin Oral Implants Res 2014;25:859-866.

42. Urban IA, Monje A, Nevins M, Nevins ML, Lozada JL, Wang HL. Surgical management of significant maxillary anterior vertical ridge defects. Int J Periodont Restorat Dent 2016;36:329-337.

43. Simion M, Fontana F, Rasperini G, Maiorana C. Vertical ridge augmentation by expanded-polytetrafluoroethylene membrane and a combination of intraoral autogenous bone graft and deproteinized anorganic bovine bone (Bio Oss). Clin Oral Implants Res 2007;18:620-629.

44. Schwarz F, Sahm N, Iglhaut G, Becker J. Impact of the method of surface debridement and decontamination on the clinical outcome following combined surgical therapy of periimplantitis: a randomized controlled clinical study. J Clin Periodontol 2011;38:276-284.

45. Monje A, Pons R, Roccuzzo A, Salvi GE, Nart J. Reconstructive therapy for the management of peri-implantitis via submerged guided bone regeneration: a prospective case series. Clinical Implant Dentistry and Related Research 2020;22:342-350.

46. Roos-Jansaker AM, Renvert H, Lindahl C, Renvert S. Submerged healing following surgical treatment of peri-implantitis: a case series. J Clin Periodontol 2007;34:723-727.

47. Schwarz F, Jepsen S, Herten M, Sager M, Rothamel D, Becker J. Influence of different treatment approaches on non-submerged and submerged healing of ligature induced peri-implantitis lesions: an experimental study in dogs. J Clin Periodontol 2006;33:584-595.

48. Roos-Jansaker AM, Renvert H, Lindahl C, Renvert S. Surgical treatment of peri-implantitis using a bone substitute with or without a resorbable membrane: a prospective cohort study. $J$ Clin Periodontol 2007;34:625-632

\section{SUPPORTING INFORMATION}

Additional supporting information may be found online in the Supporting Information section at the end of the article.

How to cite this article: Wen S-C, Barootchi S, Huang W-X, Wang H-L Surgical reconstructive treatment for infraosseous peri-implantitis defects with a submerged healing approach: A prospective controlled study. J Periodontol. 2021;1-13. https://doi.org/10.1002/JPER.21-0161 